\title{
Strain-rate effects associated with the HJC concrete model
}

\author{
Gordon Johnson ${ }^{1, *}$, Timothy Holmquist ${ }^{1}$, and Charles Gerlach ${ }^{1}$ \\ ${ }^{1}$ Southwest Research Institute, 5353 Wayzata Blvd., Minneapolis, MN, 55416, USA
}

\begin{abstract}
The Holmquist-Johnson-Cook (HJC) model for concrete was presented in 1993 and has been used extensively since that time. Since then a third invariant effect has been added and the shear modulus has been revised to vary such that Poisson's ratio is held constant. It has always been difficult, however, to determine the appropriate constant for the strain-rate effect as most of the published data are for the net stress as a function of the strain rate. Because concrete is both pressure dependent and strain-rate dependent, it is necessary to separate the individual effects. Recently strain-rate data for three concrete materials were presented by Piotrowska and others $[1,2]$, where the data are presented as equivalent stress versus confining pressure for a high strain rate and a quasi-static strain rate. This is the form necessary to determine the appropriate strain-rate effect, and the data show that the strain-rate effect is larger than used in the initial publication of the HJC model, and also that the strain-rate effect is a function of the confining pressure. For lower pressures the strain-rate effect is a factor to be applied to the quasi-static data (which is the effect represented in the original HJC model), but for higher pressures the strain rate effect is better represented by an additive term. With the addition of an another HJC constant (the pressure at which the strain rate effect transitions from a multiplied factor to an additive term) it is possible to more accurately represent the response of concrete under high pressures and high strain rates, and it is possible to compute more accurate results for projectile penetration into concrete targets. The paper presents the modified form of the HJC model, an analysis of the strain-rate effects, and results of penetration computations that are compared to experimental data in the literature.
\end{abstract}

\section{Introduction}

Concrete is a material that is difficult to model accurately under the conditions of large strains, high strain rates and high pressures. When compared to other materials, such as metals and ceramics, it has a relatively low strength and therefore can be subjected to accurate quasi-static testing under a wide range of stresses and strains. The issues in accurately determining the strength as a function of the pressure, damage and strain rate, are that these three effects are coupled together, and it is necessary to determine the effects of each if an accurate model is to be developed. An additional complexity is that there is not a standard concrete. Although concrete is often defined by its unconfined compressive strength $\left(f_{c}^{\prime}\right)$, there are wide ranges of behavior for different concrete materials with similar unconfined compressive strengths. The size and composition of the aggregate, the chemistry of the cement, the water/cement ratio, and the age, are all factors that affect the strength and failure characteristics of the concrete.

The Holmquist-Johnson-Cook (HJC) computational model for concrete was presented in 1993 [3] and it has been used extensively since then. Although it is a relative simple model, it represents the strength as a function of the pressure, damage and strain rate; the damage as a function of the pressure, the equivalent plastic strain and the volumetric plastic strain; and the pressure as a function of the

\footnotetext{
*e-mail: gordon.johnson@swri.org
}

path-dependent volumetric strain, which includes crushing. Since then a third invariant effect has been added by others $[4,5]$, and the authors have also added the third invariant option and a variable shear modulus to the HJC model in the EPIC code [6].

In the original publication of the HJC model a small strain-rate effect was determined from test data at different strain rates, but for very low pressures. Recently, experimental strain-rate data have been presented for several different concrete materials [1,2], and the data are for a range of pressures. These data are exactly what are needed to determine an improved description of the strain-rate effect in concrete. They show that the strain-rate effect is greater than previously determined for the original HJC model, and that for lower pressures the strain-rate effect is a factor to be applied to the quasi-static data (which is the effect represented in the original HJC model), but for higher pressures the strain rate effect is better represented by an additive term. The remainder of this article briefly describes the original and modified HJC models; it provides an analysis of the strain-rate data presented by Piotrowska et al. [2]; it determines the appropriate strain-rate parameters for a $48 \mathrm{MPa}$ concrete provided by Hanchak et al. [7]; it provides computed results for the response of penetrators impacting concrete targets; and it provides comparisons of these results to test data that are also presented by Hanchak et al. 


\section{The original and modified HJC model}

As this article focuses only on the strain-rate effect, the original reference [3] must be consulted for a thorough description of the entire HJC model. For the modified model an optional parameter $\left(P_{s r}^{*}=P_{s r} / f_{c}^{\prime}\right)$ has been added to allow the strain-rate effect to be additive for pressures greater than $P_{s r}$, rather than a multiplied factor. For the original model the normalized equivalent stress is given by

$$
\sigma^{*}=\left[\sigma / f_{c}^{\prime}\right]=\left[A(1-D)+B\left(P^{*}\right)^{n}\right]\left[1+C \ln \dot{\varepsilon}^{*}\right]
$$

where $A, B, n, C$ are constants, $\sigma$ is the von Mises equivalent stress (allowable strength), $D$ is the damage $(0 \leq D \leq 1), P^{*}=P / f_{c}^{\prime}$ is the normalized pressure, and $\dot{\varepsilon}^{*}=\dot{\varepsilon} / \dot{\varepsilon}_{0}$ is the normalized equivalent strain rate with $\dot{\varepsilon}$ being the actual strain rate and $\dot{\varepsilon}_{0}$ being a user input reference strain rate. The strength at $\dot{\varepsilon}_{0}$ is dependent on the pressure and the damage, and it is multiplied by the strainrate factor, $\left[1+C \ln \dot{\varepsilon}^{*}\right]$.

The modified model uses the same form for pressures less than a reference pressure $\left(P \leq P_{s r}\right)$, but for higher pressures $\left(P>P_{s r}\right)$ the strain rate effect is added to the pressure and damage dependent strength at a reference strain rate of $\dot{\varepsilon}_{0}$, as shown in Equation 2.

$$
\begin{aligned}
\sigma^{*} & =\left[A(1-D)+B\left(P^{*}\right)^{n}\right] \\
& +\left[A(1-D)+B\left(P_{s r}^{*}\right)^{n}\right]\left[C \ln \dot{\varepsilon}^{*}\right]
\end{aligned}
$$

\section{Analysis of the strain-rate data}

Piotrowska and Forquin [1] and Piotrowska et al. [2] recently presented experimental data for several different concrete materials subjected to a large range of strain rates and confining pressures. For this article the data for "siliceous aggregate" concrete [2] are analyzed. Table 1 provides data taken from Figure 15 (b) of reference [2]. The quasi-static and dynamic data from Table 1 and reference [2] are plotted as the black lines (test data) on the left side of Figure 1.

The test data provide pairs of stresses for two different strain rates at the same pressure, and this provides a means to compute effective strain-rate constants for each pair of data points. $C_{1}$ in Table 1 provides the strain-rate constants that match the test data using the original HJC strain-rate formulation and a reference strain rate of $\dot{\varepsilon}_{0}=1.0 \mathrm{~s}^{-1}$, and $C_{2}$ provides the strain-rate constants that match the test data using the original strain-rate formulation and a reference strain rate of $\dot{\varepsilon}_{0}=0.0014 s^{-1}$. These strain-rate constants can be determined in a straightforward manner using the relationship, $\sigma(\dot{\varepsilon})=\sigma\left(\dot{\varepsilon}_{0}\right)\left(1+C \ln \dot{\varepsilon}^{*}\right)$. Substituting two data sets for $\sigma(\dot{\varepsilon})$ and $\dot{\varepsilon}^{*}$ allows the two unknowns $\left[\sigma\left(\dot{\varepsilon}_{0}\right)\right.$ and $\left.C\right]$ to be determined. The third strain-rate constant $\left(C_{3}\right)$ is determined from Equation 2 for the modified model, using $P_{s r}=100 \mathrm{MPa}$. The constants for these three different conditions are shown on the right side of Figure 1.
The most dramatic observation from Figure 1 is that the effective strain-rate constants vary considerably over the range of pressures. Also, the effective strain-rate constant depends on the reference strain rate $\left(\dot{\varepsilon}_{0}\right)$. In the past the authors have generally determined the strain-rate constant for a reference strain rate of $\dot{\varepsilon}_{0}=1.0 \mathrm{~s}^{-1}$ as this allows for a more meaningful comparison of strain-rate effects for different materials. However, for the modified model it is more straightforward to have $\dot{\varepsilon}_{0}$ be the strain rate at which the quasi-static data were obtained. Finally, the constants for the modified model are less influenced by the pressure.

Looking back at the left side of Figure 1 the computed responses of the original model and the modified model are shown for two different values of $C$; the value determined from the first data point at $P=100 \mathrm{MPa}$ and an average value of $C$. These two conditions are included as it is not always possible to have a full data set for the specific concrete of interest. In fact, it is very unlikely that a full set of data (enough to determine all the constants for any concrete model) will be available for a concrete of interest. The modified model provides much better agreement with the test data, and an average value provides better agreement than the value determined from the first data point. Using the average values, the errors $\left[\left(\sigma_{\text {model }}-\sigma_{\text {test }}\right) / \sigma_{\text {test }}\right]$ for the original model range from $-22.4 \%$ to $8.5 \%$ (for $\mathrm{P}$ $=100 \mathrm{MPa}$ to $700 \mathrm{MPa}$ ), and the errors for the modified model range from $4.6 \%$ to $-2.9 \%$.

\section{Application of the modified model for projectile penetation/performation computations}

Hanchak et al. [7] provided laboratory test data (equivalent stress versus pressure) for a specific concrete (48 $\mathrm{MPa}$ ), and also provided ballistic test data (residual velocity versus striking velocity) for projectile impacts into concrete targets (for the same concrete that the equivalent stress data were provided). The left side of Figure 2 shows the laboratory test data and the fit to the data using the modified HJC model (with $P_{s r}=100 \mathrm{MPa}$ ). The test data are for quasi-static conditions and it was assumed that the strain rate for these data was $\dot{\varepsilon}=10^{-4} s^{-1}$. These data are analogous to the quasi-static test data provided in Table 1 and Figure 1. Unfortunately, no elevated strain-rate data were provided by Hanchak et al. Also included in Figure 2 are the computed responses for an increased strain rate of $10^{4} s^{-1}$, based on a strain-rate constant determined to match the ballistic test data. It should be noted that it is necessary to include the effect of the strain rate because using the quasi-static data to define the response of the concrete would result in a target resistance that is much too soft. Figure 3 shows some computed responses (with damage distributions) for projectile impact into concrete targets, using the projectile and target geometries provided by Hanchak et al. The right side of Figure 2 shows a comparison of the ballistic test data and computed results for residual velocity versus striking velocity. The computations were performed with the EPIC code [6] and they 
Table 1. Experimental data from Piotrowska et al. [2] and computed strain-rate constants.

\begin{tabular}{|c|c|c|c|c|c|c|c|}
\hline & \multicolumn{2}{|c|}{ Quasi-static data } & \multicolumn{2}{c|}{ Dynamic data } & \multicolumn{3}{|c|}{ Computed strain-rate constants } \\
\hline $\mathrm{P}$ & $\sigma$ & $\dot{\varepsilon}$ & $\sigma$ & $\dot{\varepsilon}$ & $C_{1}$ & $C_{2}$ & $C_{3}$ \\
\hline$(\mathrm{MPa})$ & $(\mathrm{MPa})$ & $\left(s^{-1}\right)$ & $(\mathrm{MPa})$ & $\left(s^{-1}\right)$ & $\dot{\varepsilon}_{0}=1.0 s^{-1}$ & $\dot{\varepsilon}_{0}=0.0014 s^{-1}$ & $\dot{\varepsilon}_{0}=0.0014 s^{-1}$ \\
\hline 0 & 0 & - & 0 & - & - & - & - \\
\hline 100 & 102 & 0.0014 & 174 & 240 & 0.0423 & 0.0586 & 0.0586 \\
\hline 200 & 180 & 0.0014 & 247 & 208 & 0.0259 & 0.0313 & 0.0552 \\
\hline 300 & 247 & 0.0014 & 318 & 177 & 0.0211 & 0.0245 & 0.0593 \\
\hline 400 & 308 & 0.0014 & 382 & 145 & 0.0183 & 0.0208 & 0.0628 \\
\hline 500 & 364 & 0.0014 & 439 & 113 & 0.0163 & 0.0182 & 0.0651 \\
\hline 600 & 415 & 0.0014 & 500 & 82 & 0.0166 & 0.0187 & 0.0759 \\
\hline 700 & 467 & 0.0014 & 553 & 50 & 0.0157 & 0.0176 & 0.0804 \\
\hline 800 & 515 & 0.0014 & - & - & - & - & - \\
\hline \multicolumn{2}{|c|}{ Averages } & 0.0014 & - & 145 & 0.0223 & 0.0271 & 0.0653 \\
\hline
\end{tabular}
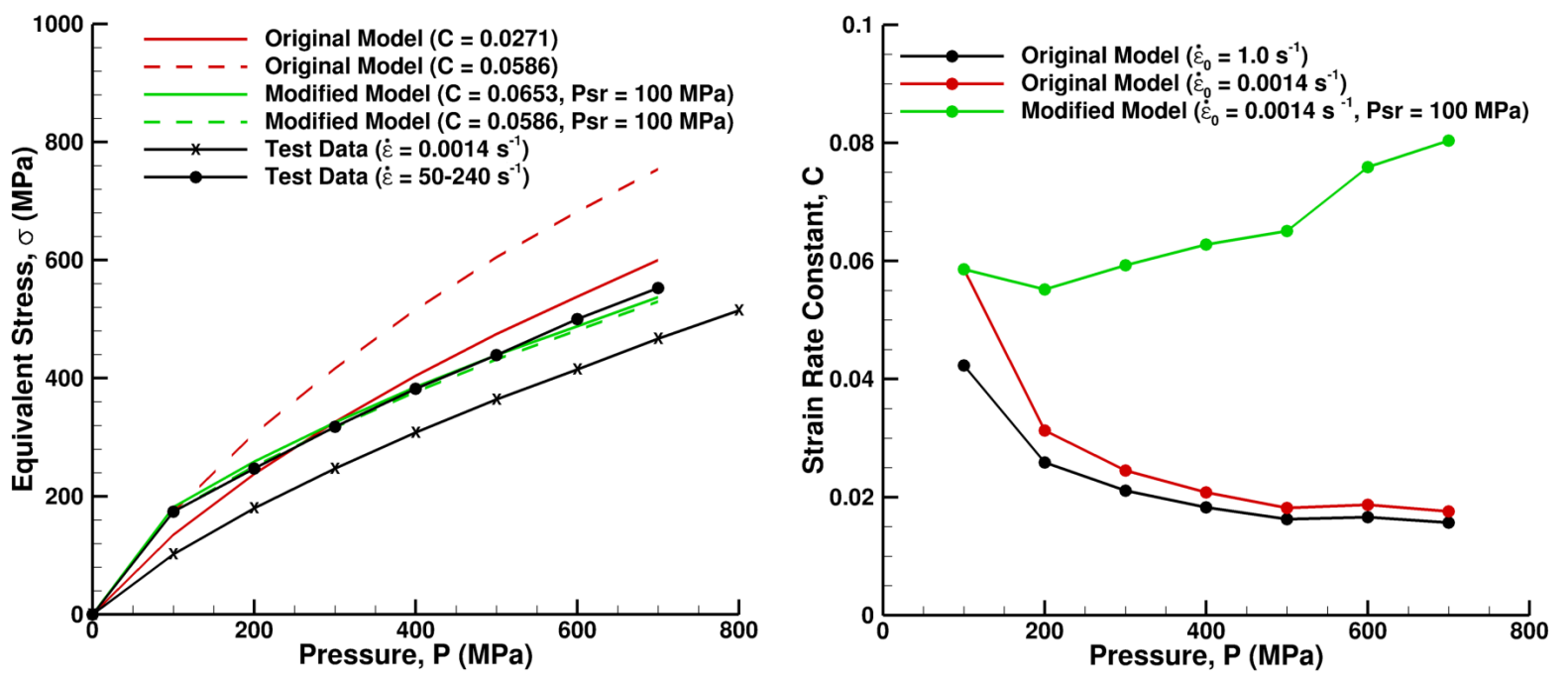

Fig. 1. Equivalent stress (left) and strain-rate constants (right) as a function of the confining pressure.

were performed in axisymmetric geometry. The Hanchak et al. tests had reinforcing steel in the target and this was also included in the computations. There is very good agreement between the test data and the computed results.

Although the agreement between the test data and the computed results is encouraging, there are some other factors that must be considered. First, it appears that there is no universal strain-rate constant for a range of concrete materials. The publications by Piotrowska and Forquin [1] and Piotrowska et al. [2] examined several different types of concrete, and the strain-rate constants were not consistent for the different materials. Most of the materials behaved in a manner similar to that considered here (with the modified model), but one of the materials (with limestone aggregate) behaved in a manner more like the original model. The one thing they all had in common is that the strain-rate effect is significant, and that it cannot be ignored for high-strain-rate computations. The strain-rate constant used to match the penetration/perforation results (in Figures 2 and 3 ) is $C=0.027$, and this is significantly lower than those determined from the high-strain-rate data considered here [2]. It is, however, much higher than the $C=0.007$ published by Holmquist et al. in 1993 [3]. Fur- thermore, when the computations are used to determine the strain-rate constant, that constant not only includes the strain-rate effect, but it also includes all the other errors in the computation. There are errors associated with 2D verses $3 \mathrm{D}$ effects, the numerical algorithm (which used an erosion strain of 2.5), the form of the material model (including strength, pressure, damage, third invariant), and the many other constants in the model. All of these potential errors are incorporated into the strain-rate constant which provides a match to the data. Even with these concerns, if a full data set of experiments is not available for a concrete of interest, then the approach presented here may be the best alternative. An encouraging result is that the strain-rate constant is within the range of strain-rate effects experienced in the laboratory tests.

\section{Summary and conclusions}

This article has taken high strain-rate data from the literature and developed an optional formulation for the strainrate effect in the HJC computational model for concrete. The strain-rate effect is significant and highly dependent on the confining pressure. For lower pressures the strain- 

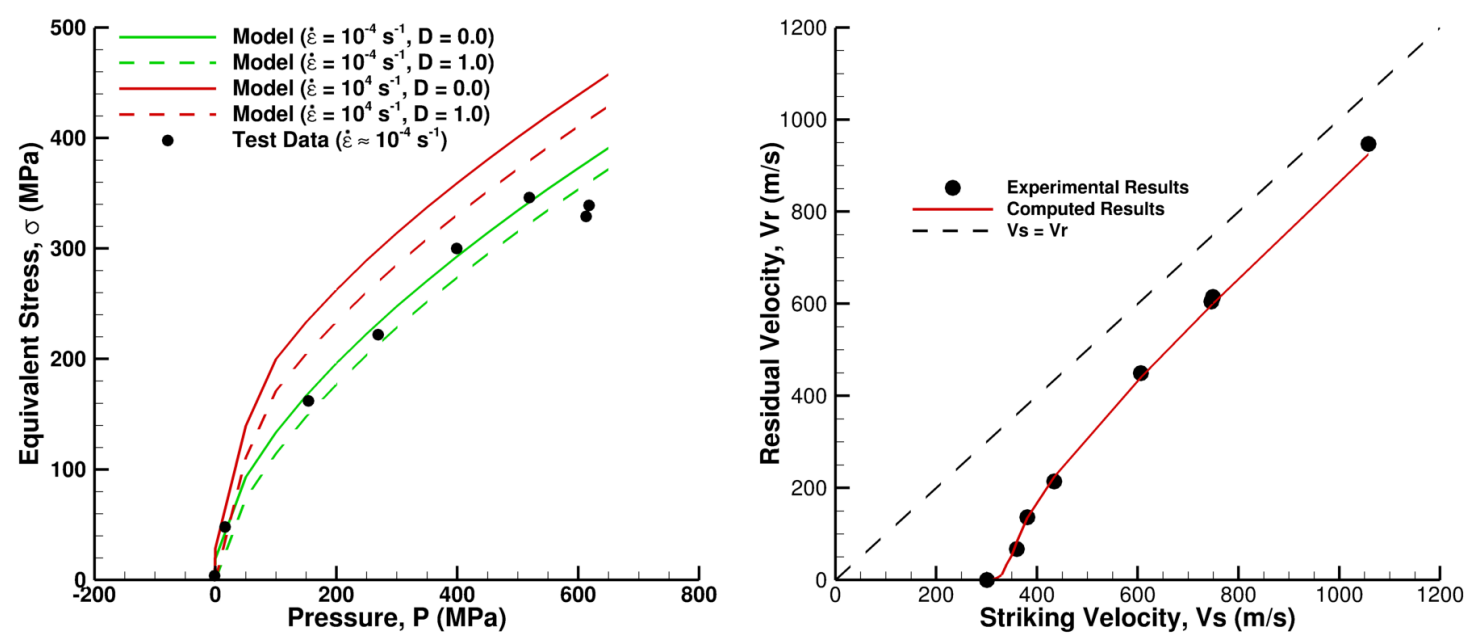

Fig. 2. Comparisons of model and laboratory test data for equivalent stress versus pressure (left); and computed ballistic results and test data for residual versus striking velocity.

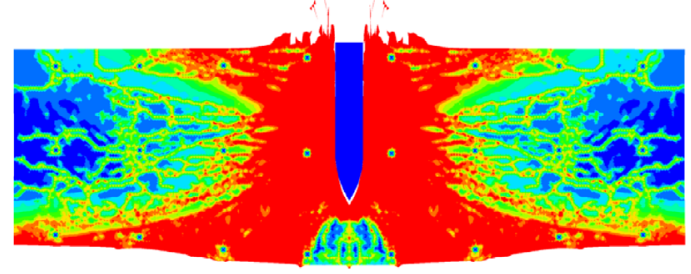

$\mathrm{V}_{\mathrm{s}}=301 \mathrm{~m} / \mathrm{s}$

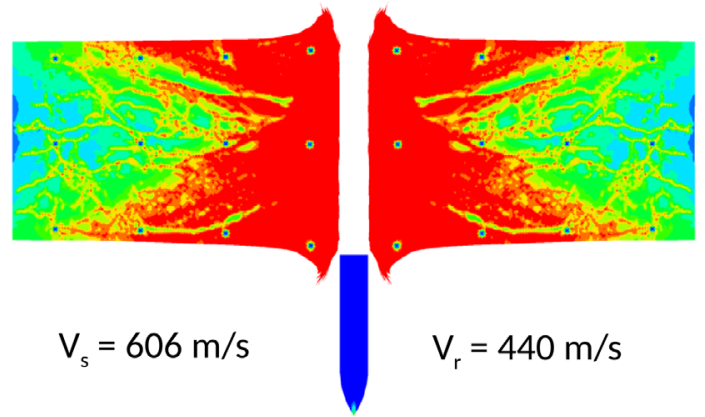

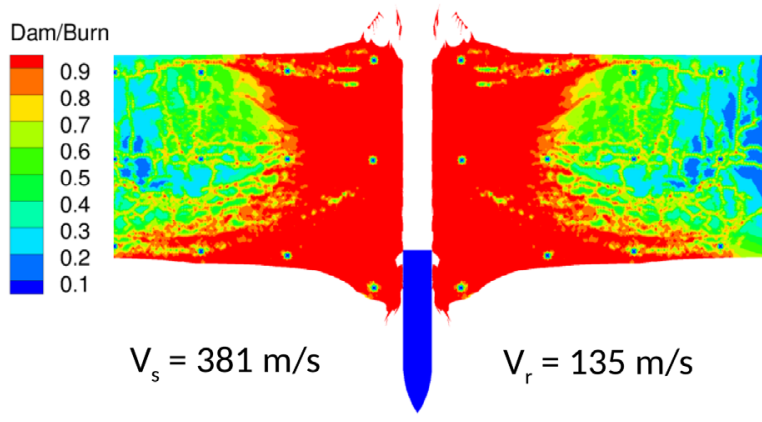

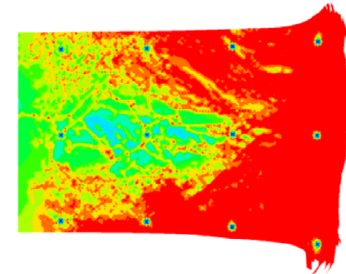

$\mathrm{V}_{\mathrm{s}}=1058 \mathrm{~m} / \mathrm{s}$

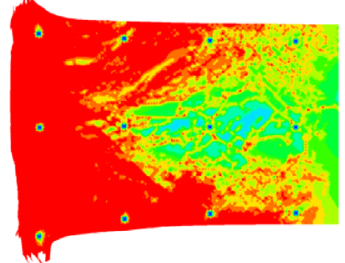

$V_{r}=922 \mathrm{~m} / \mathrm{s}$

Fig. 3. Computed responses of a projectile impacting a concrete target at different velocities. The projectile is steel, with a length of $144 \mathrm{~mm}$, a diameter of $25.4 \mathrm{~mm}$ and a mass of $0.50 \mathrm{~kg}$. The thickness of the target is $178 \mathrm{~mm}$.

rate effect is a factor to be applied to the quasi-static data (which is the form provided in the original HJC model), but for higher pressures the strain-rate effect is better represented by an additive term. It has been shown that the modified form of the model provides better agreement with published test data from Piotrowska et al. [2]. The modified model has also been used to perform computations of projectile impacts into concrete targets. Here the strain-rate constant was determined from the projectiletarget test data, but it provided very good agreement between the experimental data and the computed results for a wide range of impact velocities. A continuing issue associated with computations involving concrete (subjected to large strains, high strain rates and high pressures) is that there are many different types of concrete, and that many different laboratory tests are required to obtain the data to thoroughly characterize a single material. As a result most computations must be performed with less than a full set of independently-determined constants.

This work was performed under contract W15QKN-14-9-1001. The authors would like to thank Capt. Ralph Tatum and Dr. Angela Diggs (Air Force Research Laboratory) for their contributions to this work. 


\section{References}

1. E. Piotrowska, P. Forquin, Journal of Dynamic Behavior of Materials (2015)

2. E. Piotrowska, P. Forquin, Y. Malecot, Mechanics of Materials 92 (2016)

3. T.J. Holmquist, G.R. Johnson, W. Cook, A Computational Constitutive Model for Concrete Subjected to Large Strains, High Strain Rates and High Pressures, in Fourteenth International Symposium on Ballistics (Quebec City, Canada, 1993)
4. A.O. Frank, M.D. Adley, On the Importance of a Three-Invariant Model for Simulating the Perforation of Concrete Slabs, in Seventy-Eighth Shock and Vibration Symposium (Philadelphia, Pennsylvania, 2007)

5. P. Polanco-Loria, O.S. Hopperstad, T. Borvik, T. Berstad, International Journal of Impact Engineering 35 (2008)

6. G.R. Johnson, International Journal of Impact Engineering 38 (2011)

7. S.J. Hanchak, M.J. Forrestal, E.R. Young, J.Q. Ehrgott, International Journal of Impact Engineering 12 (1992) 
